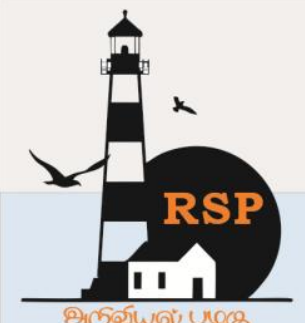

INTERNATIONAL RESEARCH JOURNAL ON ADVANCED SCIENCE HUB

RSP SCIENCE HUB

(The Hub of Research Ideas)

Available online at www.rspsciencehub.com

\title{
Machine learning amalgamation of Mathematics, Statistics and Electronics
}

Trupti S. Gaikwad ${ }^{1}$, Snehal A. Jadhav ${ }^{2}$, Ruta R. Vaidya ${ }^{3}$, Snehal H. Kulkarni ${ }^{4}$

1,2,3,4 Trupti S. Gaikwad, Dept. of Computer Science, Vishwakarma College of Arts, Commerce and Science, Maharashtra, India

trupti08jadhav@gmail.com ${ }^{1}$

\section{Abstract}

Interdisciplinary research is a manner of research carried out by an individual or group of persons. The knowledge, data, techniques, concepts are incorporated from two or more disciplines. In this paper we tried to throw light on this concept. Machine learning is a branch of computer science which uses the information, tools for collection of data, methods for analysis from the subjects like Electronics, Mathematics and Statistics. Why we use machine learning? Because it plays an influential role in prediction of data. Machine learning is used to find hidden patterns and essential ideas from data as well as it solve complex problems. In today's world, many applications have large volume of data like structured, unstructured and semi structured. This unexploited resource of knowledge can be used to improve business decisions. As data diversifies many are adapting to machine learning tool for analysis of data, so that, they can exploit intelligence and benefit from that data at most. Machine learning adopts different algorithms and each algorithm performs different functionality. In this paper, we tried to explain through example, how Electronics is used for collection of data while Mathematics and Statistics are used for analysis and finally using Machine learning results can be predicted.

Keywords: Machine learning, Analysis, Statistics, Electronics, Mathematics

\section{Introduction}

Machine learning is intersection of Mathematics, Statistics, probabilistic, Electronics, and Computer Science. Machine learning uses algorithm to learn iteratively from data and using positive feedback can build an intelligent application. Though machine learning differs from traditional computational access, it is part of computer science. In conventional programming, algorithms are explicitly written for problem solving used by computer. On other hand machine learning is a concept that a machine can learn from past experience and previously solved examples, without being explicitly programmed. For example, biometric attendance, fraud detection, face recognition, text recognition etc. 


\subsection{What is Machine Learning?}

Machine learning is the study of computer algorithms that improve automatically through experience [1-4]. It provides ability to system, learn and improve using previous experience without being explicitly programmed. It mainly focuses on the development of a program so that they can access data and learn it for themselves.

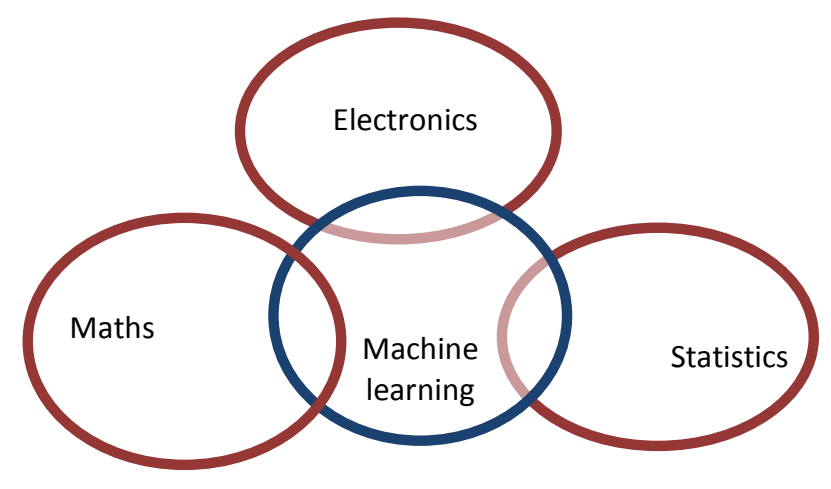

Fig.1. Interdisciplinary Machine Learning

How machine learning works?

The learning process starts with observations or data, like direct experience, example or instruction, so that it will find patterns for data and make better determination about future based examples provided. The main goal is to allow the system to learn automatically without human intervention and modify actions accordingly. It has different types of algorithm. Machine learning algorithms are classified as:

- Supervised machine learning- In supervised machine learning input variable $(\mathrm{X})$ use by an algorithm to give an output variable $(\mathrm{Y})$. Here the algorithm learns the mapping function from input to the output. The mapping function is $\mathrm{Y}$ $=f(X)$. The main aim of an algorithm is to estimate the mapping function so that for new input data $(\mathrm{X})$, the algorithm can predict the output variable (Y) for that data.

The process of learning of the algorithm from the training data set, it can be considered as a teacher supervising the learning process so that it is called supervised learning. It can be further grouped into regression and classification problem.

Examples of supervised learning algorithm are

1. For classification problemsSupport vector machines

2. For regression problem- Linear regression

3. For classification and regression problem- Radom forest.

- Unsupervised machine learningUnsupervised machine learning has only input data (X). It does not have related output variable (Y). To acquire more knowledge about the data underlying structure is designed or distribution in the data is done. It is called unsupervised learning because it does not have correct answer and teacher. Algorithms itself can discover and present the interesting structure in the data. It can further group into clustering and association problems.

Unsupervised learning algorithm uses following algorithms

1. For clustering problem-k means 
2. For association rule miningApriori algorithm

- Semi supervised machine learningIn semi supervised machine learning only for some of input variable(X), an output variable (Y) is present. It is a composite of supervised machine learning and unsupervised machine algorithm. Here we can use both

i) Supervised learning technique where we want best prediction for unlabeled data, so that we can feed that data back to supervised learning algorithm as training data and use that model to make prediction for new unseen data.

ii) Unsupervised learning technique to find and learn the structure in the input variables.

Most of today's real world problems are from this type as problems can be expensive or cheap. For expensive label problems domain experts are required and cheap unlabeled problems are easy to collect and store data.

- Reinforcement Learning- The machine is exposed to environment and it learns itself by using positive feedback and negative feedback. The machine learns from past experience. It is directed to make specific decisions. It tries to capture the best possible knowledge to make accurate business decisions.

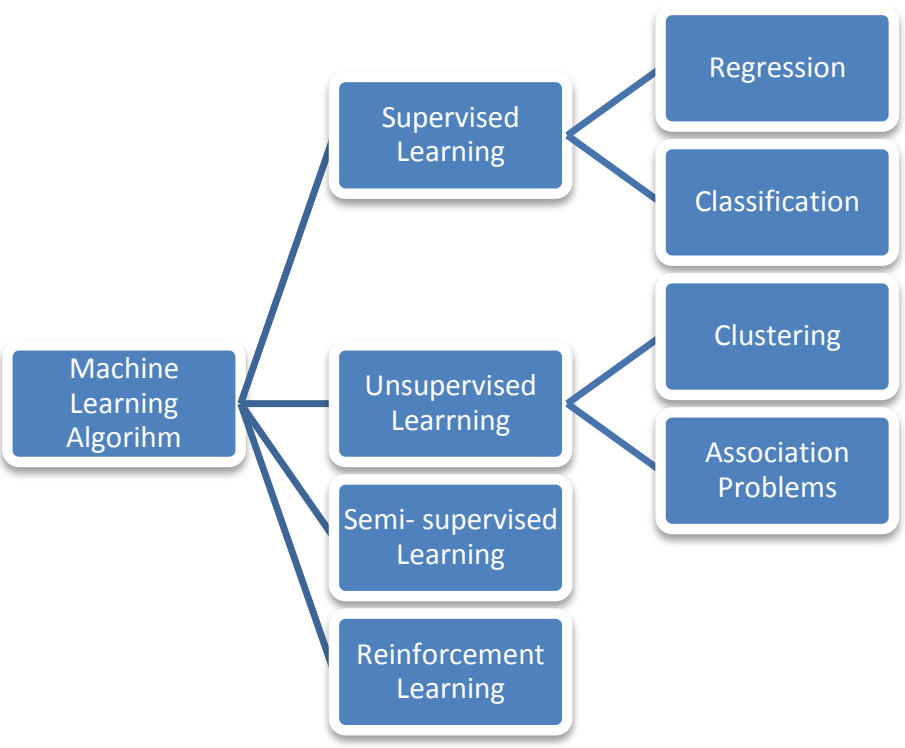

Fig.2. Classification of Machine Learning Algorithm

\subsection{Electronics: Elementary component of machine learning}

Electronics is primary element whenever we talk about automated or intelligent or smart systems. Electronics and different branches of computer science like machine learning, artificial intelligence are blended together to invent new applications. The use of machine learning in engineering field is very vital for signal processing. Due to this, there is increase in accuracy and quality when sound, images, and other inputs are transmitted. Machine learning algorithms are helpful to model signals, for pattern detection, to draw meaningful inferences, and make precise adjustments to signal output.[5-8]

To feed the data in machine learning systems signal processing techniques are useful. 
www.rspsciencehub.com

Digital signal processing and digital image processing these two are used in many applications along with machine learning.

Smart sensors are also used along with machine learning for developing number of applications like weather forecast system, in healthcare instruments, in smart home systems etc.

\subsection{Mathematics behind machine learning}

Mathematics is main part of machine learning as it is used at backend. Machine learning acquires data through algorithms and then uses this data to make predictions. Machine Learning requires mathematical knowledge. It includes linear Algebra, Calculus, Statistics, Discrete Maths, Probability and Optimization which help to create algorithms. It includes accuracy, training time, model complexity, choosing parameter setting and validation strategies. Importance of Math's topics needed for machine learning is:

Linear Algebra: It is the backbone of machine learning. To find the values of variables $\mathrm{X}$ and $\mathrm{Y}$ matrix operations are used which are parts of linear algebra. Due to this, linear algebra is necessary in machine learning. Not only all the operations in Linear Algebra are systematic rules but also structural representation of the knowledge that a computer can understand easily. Topics needed for understanding the methods of machine learning in Linear Algebra are LU decomposition, orthogonalization, matrix operations, projections, Eigen values, Eigen vectors, Vector spaces,
Volume 02 Issue 07 July 2020

Singular Value Decomposition (SVD) etc.

Natural language processing on tabular datasets, data files such as encoding and dimensionality reduction, images etc is applications of Linear Algebra in machine learning.

Multivariate Calculus: The most of machine learning algorithms are trained on multiple variables. It helps to better quantify and predict the information accurately. Laplacian and Lagragian Distribution, Vector-Values Functions, Directional Gradient, Differential and Integral Calculus, Partial Derivatives, Jacobian are the methods of Multivariate Calculus use in machine learning. Examples of Multivariate Calculus include calculation of monthly rain fall, temperature and wind speed and so on.

Graph theory: Graphs represent flow of computation. Graph learning models can be used to learn machine learning algorithms. Graphs represent computationally various matrices while matrix provides different types of information. The machine learning models having graph like structure are K-mean, K-nearest neighbours, Decision trees, Random forest, neural networks.

\subsection{Statistics: Prerequisite of machine learning}

Statistics plays important role in machine learning. Definitely statistical knowledge is applied to machine learning through predictive analysis. Following are the examples where Statistics can be applied in machine learning.

- Problem Designing

- Sympathize Data 
- Data cleansing

- Choosing Data

- Data Organization

- Model Assessment

- Model Organization

- Model Demonstration

- Model Anticipation

\section{Predictive analysis:}

As the name suggests this analysis is used to make predictions about unknown incoming events. From given data sets the information is educed to find out patterns and anticipate future trends, outcomes etc. The data source is customer service data, survey data, digital marketing data, geographical data etc. Basically here different techniques from machine learning, artificial intelligence, data mining, and Statistics are used. Generally predictive model is executed using time series regression curve and surface fitting etc. Predictive models are divided into two classes as parametric and non parametric. A predictive analytics model has three key components:

- Historical Data - Using past data predictions about the future trends are made.

- $\quad$ Statistical Modeling - Regression analysis stimulate the prediction using past data.

- Assumptions - Predictive analytics builds on the simple assumption that future trends in data are based on past trends.

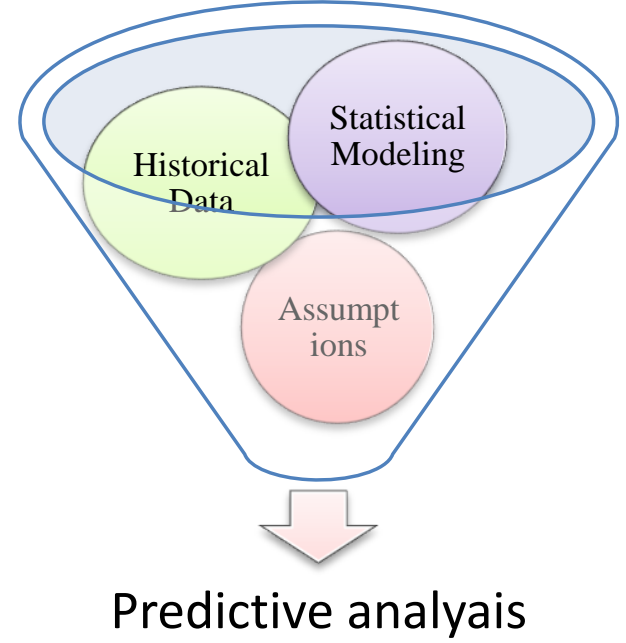

Fig.3. Predictive Analysis

\subsection{Creation of predictive model:}

Following are the steps involved.

- The outliers are removed, missing data is treated thus date is cleaned.

- The predictive model is distinguished as parametric or non parametric model.

- The data is converted into suitable format for selected modeling algorithm. The subset of data is to be specified here.

- Parameters are estimated for training data sets.

- It is checked whether chosen model is appropriate by performing goodness of fit test.

- Modeling accuracy of data is tested.

- Model is used for prediction.

We will consider some examples of different predictive models.

Classification model: This model is generally used in answering yes or no type of 
questions which on further analysis guide for conclusive action. So this model is used in industries.

Clustering model: In this model the data is sorted into different groups based on similar attributes.

Forecast model: This is frequently used model as the numeric value is estimated from the data.

Outlier model: This model identifies abnormal data figures from the data set. It is useful in retail and finance industry.

Time series model: This model consists of data having time as input parameter.

Regression model: It finds relationship between dependent and two or more independent variable. This relationship is used to predict unknown variable based on known predictions.

Decision trees: It is a tree like model of decisions and possible consequences. This is based on Boolean test. In this case problems are organized as tree with end nodes as branches.

\subsection{Probability:}

We can say that fundamental principle of Machine learning is 'Probability'. In many domains of machine learning probability theory is applied. From collected data probability helps to make decisions. These inferences are further used to predict future trends. Many techniques like maximum likelihood estimation (MLE) are based on probability theory. MLE is used in linear regression, logistic regression, artificial neural networks etc.

\subsection{Regression:}

Technique of prediction on the basis of correlation is called as regression. Further there are different regression methods. Simple linear regression is the relationship between two sets of variables. It is used to predict daily maximum temperature, while the aim of multiple regressions is to find explanatory and response variables. Previously it was used to predict weather conditions at a particular place. There are many more types of regression which can be used in applications of machine learning like weather forecasting, healthcare etc.[9-14]

\section{Applications of machine learning}

In machine learning computers, software and devices learn via cognition. Here are some most important day to day applications of machine learning.

- Virtual personal assistants-

Virtual personal assistant like Siri, Alexa, and Google are popular examples of machine learning. Here over a voice question is asked and it provides information by recalling related queries or sends a command to other electronic devices to collect information. Virtual assistants like smart speakers, smart phones, and mobile apps are used.

- Traffic prediction-

GPS navigation services are used to predict traffic. Map of current traffic is built using 
current location of vehicles and velocities which are stored at a central server which helps to handle traffic. Using machine learning the congestion can be found on the basis of daily experience.

- Video surveillance-

The video surveillance in previous days is tedious job for human attendants. Nowadays it can detect crimes before they happen by finding the unusual behavior of person. This can be tracked by system and give alarm to human attendants.

- Healthcare-

With the help of wearable sensors and devices, real time health of a patient can be used to collect patient information such as heart beat, blood pressure and other vital parameters. This type of information can be used by doctor and medical expert to analyze the health condition of an individual and can predict future ailments

- Digital photography-

In digital photography a matrix is used. Operations on the image shearing, cropping, scaling, rotation, translation are all described using the notation and operations of Linear Algebra.

These are some of the applications of machine learning. Here we are focusing on the application "Weather Forecasting".

\section{Weather Forecasting-}

The huge change in climate due to global warming has made weather forecasting trending research topic. That's why supervision and prediction of weather is very important task nowadays. Traditional techniques of weather forecasting uses big complex models of physics, in which different atmospheric conditions are monitored over a long period of time. These conditions are often unstable because of disturbance of the weather system, which results in inaccurate forecasts.

Samples from present weather are taken and by using numerical algorithms of fluid dynamics and thermodynamics the future state is predicted. These equations are nonlinear therefore numerical methods are used to find approximate solutions. Different models use different solution methods. For solving the system of ordinary differential equations which uses physical model is unstable under disturbances, and uncertainties in the initial measurements of the atmospheric conditions. So, this whole process is bit complex and causes fault weather predictions. Here we are introducing machine learning which is relatively robust to disturbances and doesn't require a complete understanding of the physical processes that govern the atmosphere. Therefore, machine learning is alternate and effective method to physical models in weather forecasting. An automatic weather station (AWS) is an automated version of the traditional weather station, in which measurements can be taken from 


\section{www.rspsciencehub.com}

either remote area without interaction of human being. Due to rapid technological development in the area of sensors and measuring system it has become very easy to read the natural parameters like temperature, humidity, rainfall by using different smart sensors like temperature sensor, humidity sensors etc. By utilizing the smart sensors for checking the weather conditions, parameters can be measured and stored accurately. These parameters can be sent to base station using wireless communication facility. After receiving the parameters from particular location the role of Mathematics and Statistics starts.

The weather forecasting is divided into two steps. The first step is mathematical modelling of the physical process happening in atmosphere. This includes Numerical Weather Predictions (NWP) based on integration of basic numerical equations. To predict the weather based current conditions use mathematical models of the atmosphere and oceans. Most atmospheric models are numerical, i.e. they discretize equations of motion. They can predict micro scale phenomena. Then in the second step, we will use these data to train simple machine learning models which can predict correct weather conditions for the next few days. It includes statistical methods like regression, predictive analysis etc. Here linear regression and a variation of functional regression these two techniques are used. The model is checked for efficiency and can improve using parameter tuning and cross validation. After that algorithm gives
Volume 02 Issue 07 July 2020 current weather condition and predicts next day's weather condition.
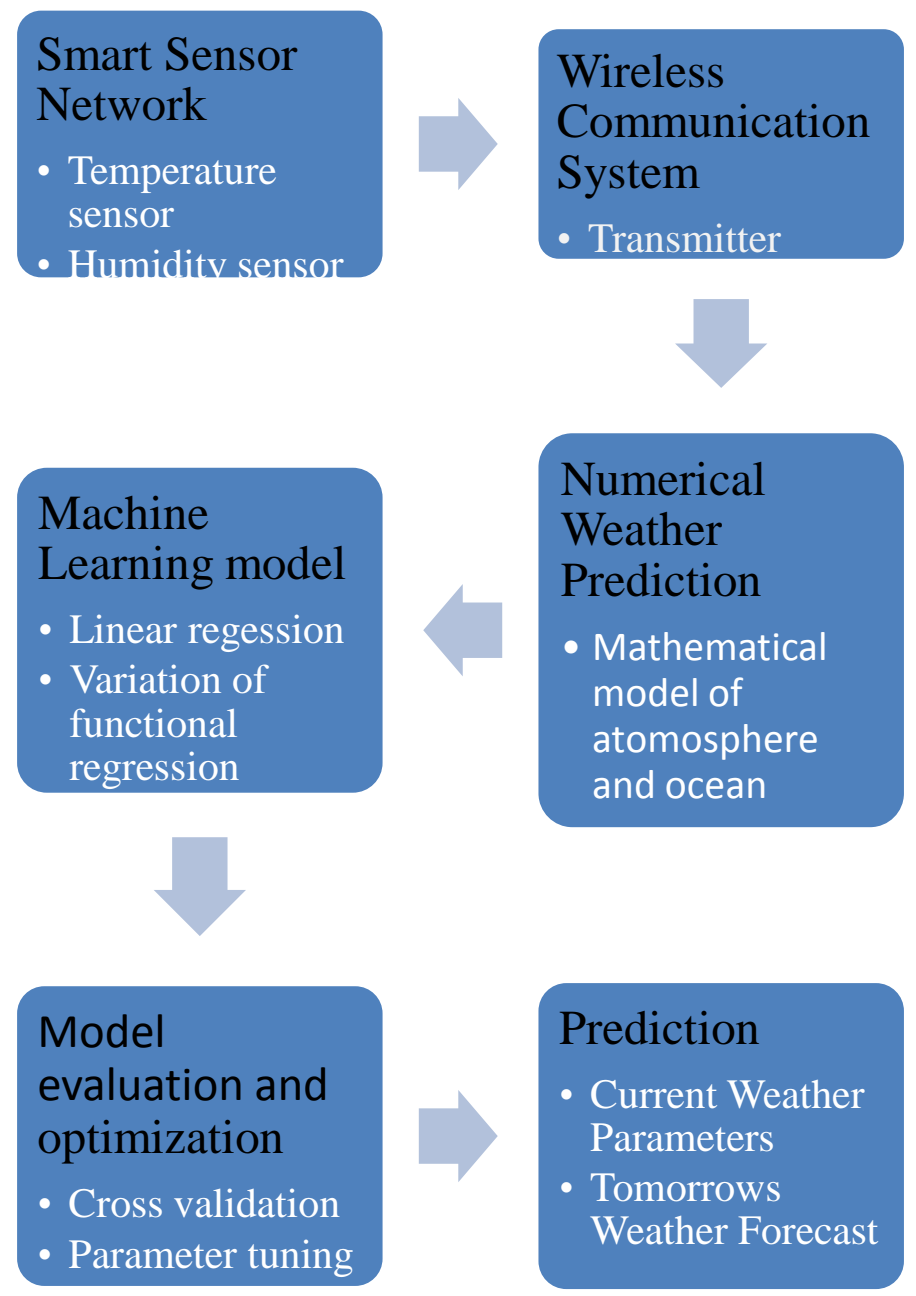

Fig.4.Weather Forecasting

\section{Conclusion:}

Machine learning is continuously revolutionary field of computer science. In today's world real time problems are solved by machine learning by storing, manipulating, extracting and retrieving data from large sources. In this paper we have focused how machine learning model can be efficiently and effectively designed using Electronics, Mathematics and Statistics. In day to day life we use many applications of machine learning like Google translator, Google directions. 
www.rspsciencehub.com

Many of us use these applications without being known how machine learning is blended with the subjects like Mathematics, Statistics and Electronics. We have discussed example of weather forecasting which is complex task to understand the capabilities of above mentioned subjects. It is important to note that the algorithms, methodologies, methods used by machine learning to solve problems will continue to change. In short machine learning has ability to learn from ideas, and adapt in changing situations with high accuracy, speed and precision.

\section{References:}

[1] https://en.wikipedia.org/wiki/Machine_lear ning

[2] https://machinelearningmastery.com/superv ised-and-unsupervised-machine-learningalgorithms/

[3] https://towardsdatascience.com/themathematics-of-machine-learning$894 \mathrm{f} 046 \mathrm{c} 568$

[4] https://medium.com/analytics-vidhya/roleof-mathematics-in-machine-learningafc $75101 f 877$

[5] https://www.newgenapps.com/blog/machin e-learning-vs-predictive-analytics/

[6] https://machinelearningmastery.com/statisti cal-methods-in-an-applied-machinelearning-project/

[7] https://www.mathworks.com/discovery/pre dictive-modeling.html
Volume 02 Issue 07 July 2020

[8] https://www.logianalytics.com/predictiveanalytics/predictive-algorithms-andmodels/

[9] https://www.conceptatech.com/blog/bestdata-science-methods-for-predictive-analytics [10] https://www.linkedin.com/pulse/regressionanalysis-weather-forecasting-chonghua-yin

[11] https://www.datasciencecentral.com/profiles/ blogs/understanding-the-applications-ofprobability-in-machine-learning

[12] https://en.wikipedia.org/wiki/Automatic_weat her_station

[13] https://en.m.wikipedia.org/wiki/Atmospheric _model

[14] https://www.analyticsvidhya.com/blog/2017/ 09/common-machine-learning-algorithms/ 